\title{
Stability of Attractive Bose-Einstein Condensates in a Periodic Potential
}

\author{
J. C. Bronski ${ }^{1}$, L. D. Carr ${ }^{2}$, R. Carretero-González ${ }^{3}$ B. Deconinck ${ }^{4}$, J. N. Kutz 4 , and K. Promislow ${ }^{3}$ \\ ${ }^{1}$ Department of Mathematics, University of Illinois Urbana-Champaign, Urbana, IL 61801, USA \\ ${ }^{2}$ Department of Physics, University of Washington, Seattle, WA 98195-1560, USA \\ ${ }^{3}$ Department of Mathematics, Simon Fraser University, Burnaby, B.C., CANADA V5A $1 S 6$ \\ ${ }^{4}$ Department of Applied Mathematics, University of Washington, Seattle, WA 98195-2420, USA
}

\begin{abstract}
Using a standing light wave trap, a stable quasi-one-dimensional attractive dilute-gas BoseEinstein condensate can be realized. In a mean-field approximation, this phenomenon is modeled by the cubic nonlinear Schrödinger equation with attractive nonlinearity and an elliptic function potential of which a standing light wave is a special case. New families of stationary solutions are presented. Some of these solutions have neither an analog in the linear Schrödinger equation nor in the integrable nonlinear Schrödinger equation. Their stability is examined using analytic and numerical methods. Trivial-phase solutions are experimentally stable provided they have nodes and their density is localized in the troughs of the potential. Stable time-periodic solutions are also examined.
\end{abstract}

\section{INTRODUCTION}

$$
i \psi_{t}=-\frac{1}{2} \psi_{x x}-|\psi|^{2} \psi+V(x) \psi
$$

Dilute-gas Bose-Einstein condensates (BECs) have been generated by many groups using different gases which are cooled to very low temperatures and confined in magnetic fields or standing light waves. The sign of the atomic coupling determines whether the interaction of the BECs is repulsive or attractive. Note that efficient tuning between attractive and repulsive condensates can be achieved via a Feshbach resonance [1]. Repulsive BECs are experimentally stable [2]. In contrast, attractive Lithium BECs have been shown to collapse in three dimensions 3 5, but are predicted to be stable in quasi-one-dimension [6]. Here, we study the dynamics and stability of quasi-one--dimensional, attractive BECs trapped in standing light waves.

The mean-field description for the macroscopic BEC wavefunction is constructed using the Hartree-Fock approximation [7], resulting in the Gross-Pitaevskii equation [8,9]. The quasi-one-dimensional regime of the Gross-Pitaevskii equation holds when the transverse dimensions of the condensate are on the order of its healing length and the longitudinal dimension is much longer than its transverse dimensions [6, 10]. In this regime the $\mathrm{BEC}$ remains phase coherent and the governing equations are one-dimensional. This is in contrast to a truly 1D mean-field theory which requires transverse dimensions on the order of or less than the atomic interaction length [11]. In quasi-1D, the Gross-Pitaevskii equation reduces to the cubic nonlinear Schrödinger equation (NLS) with a potential [6,12,13].

In this paper we construct new solutions corresponding to a quasi-one-dimensional attractive BEC trapped in an external periodic potential. The governing equation is given by the nonlinear Schrödinger equation with a potential where $\psi(x, t)$ represents the macroscopic wave function of the condensate and $V(x)$ is an experimentally generated macroscopic potential. A large class of periodic potentials is given by

$$
V(x)=-V_{0} \operatorname{sn}^{2}(x, k)
$$

where $\operatorname{sn}(x, k)$ denotes the Jacobian elliptic sine function 114 with elliptic modulus $0 \leq k \leq 1$. In the limit $k \rightarrow 1^{-}, V(x)$ becomes an array of well-separated hyperbolic secant potential barriers or wells, while in the limit $k \rightarrow 0^{+}$it becomes purely sinusoidal. We note that for most intermediate values (e.g. $k=1 / 2$ ) the potential closely resembles sinusoidal behavior and thus provides a good approximation to a standing wave potential. The potential is plotted in Fig. 1 for values of $k=0,0.9,0.999$ and 0.999999. Only for $k$ very near unity (e.g. $>0.999$ ) does the solution start to appear visibly elliptic. The freedom in choosing $k$ allows great flexibility in considering a wide variety of physically realizable periodic potentials.

The paper is outlined as follows: in the next section we derive and consider various properties and limits of two types of explicit solutions of Eq. (1) with (2). Section III develops the analytic framework for the linear stability properties of the new solutions of Sec. II. The stability results are confirmed by numerical computations. For many cases, the stability analysis yields only partial analytical results, and we rely on numerical experiments to determine stability. Nonstationary solutions are discussed in Sec. IV and illustrated with various types of time-periodic solutions. We conclude the paper in Sec. V with a brief summary of the primary results of the paper and their consequences for the dynamics of an attractive BEC. 


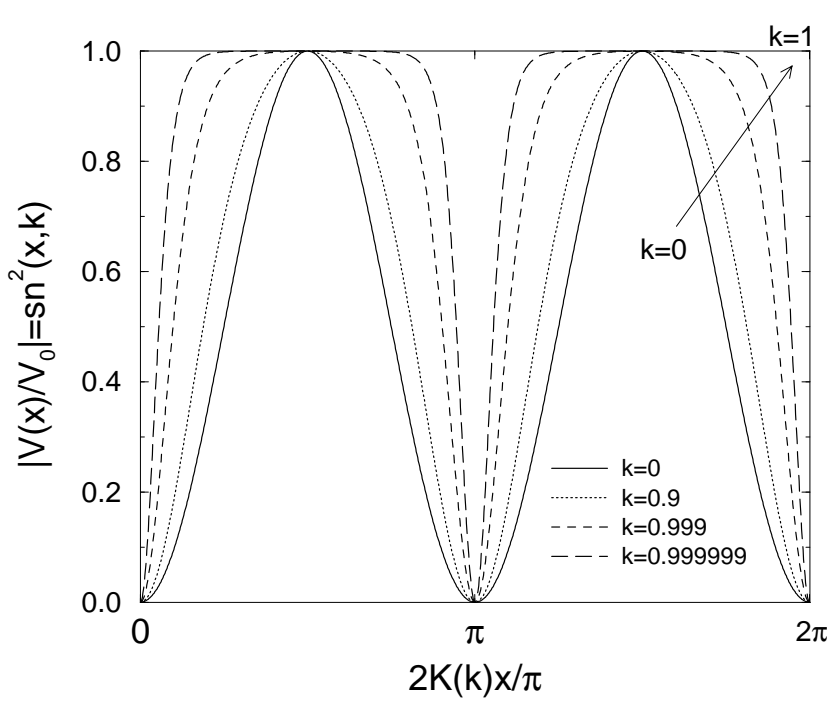

FIG. 1. The $\operatorname{sn}^{2}(x, k)$ structure of the potential for varying values of $k$. Note that the $x$-coordinate has been scaled by the period of the elliptic function. Since $\operatorname{sn}(x, k)$ is periodic in $x$ with period $4 K(k)=4 \int_{0}^{\pi / 2} d \alpha / \sqrt{1-k^{2} \sin ^{2} \alpha}, V(x)$ is periodic in $x$ with period $2 K(k)$. This period approaches infinity as $k \rightarrow 1$.

\section{STATIONARY SOLUTIONS}

Equation (11) with $V(x)=0$ is an integrable equation and many explicit solutions corresponding to various boundary conditions are known. A comprehensive overview of these solutions is found in 15 . If $V(x) \neq 0$, NLS is not integrable. In this case, only small classes of explicit solutions can most likely be obtained. Our choice of potential (2) is motivated by the form of the stationary solution of the NLS with $V(x)=0$. An overview of these stationary solutions and their properties is found in [10]. At present, we restrict our attention to stationary solutions of Eq. (1), i.e., solutions whose time-dependence is restricted to

$$
\psi(x, t)=r(x) \exp (-i \omega t+i \theta(x)) .
$$

If $\theta_{x} \equiv 0$, then the solution is referred to as having trivial phase and we choose $\theta(x)=0$. Substituting the ansatz Eq. (3) in Eq. (1) and dividing out the exponential factor results in two equations: one from the real part and one from the imaginary part. The second equation can be integrated:

$$
\theta(x)=c \int_{0}^{x} \frac{d x^{\prime}}{r^{2}\left(x^{\prime}\right)},
$$

where $c$ is a constant of integration. Note that $\theta(x)$ is a monotonous function of $x$. Substitution of this result in the remaining equation gives

$$
\omega r^{4}(x)=\frac{c^{2}}{2}-\frac{r^{3}(x) r^{\prime \prime}(x)}{2}-r^{6}(x)-V_{0} \operatorname{sn}^{2}(x, k) r^{4}(x) .
$$

The following subsections describe two classes of solutions of this equation.

\section{Type A}

\section{Derivation}

For these solutions, $r^{2}(x)$ is a quadratic function of $\operatorname{sn}(x, k)$ :

$$
r^{2}(x)=A \operatorname{sn}^{2}(x, k)+B .
$$

Substituting this ansatz in Eq. (5) and equating the coefficients of equal powers of $\operatorname{sn}(x, k)$ results in relations among the solution parameters $\omega, c, A$ and $B$ and the equation parameters $V_{0}$ and $k$. These are

$$
\begin{aligned}
\omega & =\frac{1}{2}\left(1+k^{2}-3 B+\frac{B V_{0}}{V_{0}+k^{2}}\right), \\
c^{2} & =B\left(\frac{B}{V_{0}+k^{2}}-1\right)\left(V_{0}+k^{2}-B k^{2}\right), \\
A & =-\left(V_{0}+k^{2}\right) .
\end{aligned}
$$

For a given potential $V(x)$, this solution class has one free parameter $B$ which plays the role of a constant background level or offset. The freedom in choosing the potential gives a total of three free parameters: $V_{0}, k$ and $B$.

The requirements that both $r^{2}(x)$ and $c^{2}$ are positive imposes conditions on the domain of these parameters:

$$
\begin{gathered}
V_{0} \leq-k^{2}, \quad B \geq 0, \quad \text { or } \\
V_{0} \geq-k^{2}, \quad\left(V_{0}+k^{2}\right) \leq B \leq\left(1+\frac{V_{0}}{k^{2}}\right) .
\end{gathered}
$$

The region of validity of these solutions is displayed in Fig. 2.

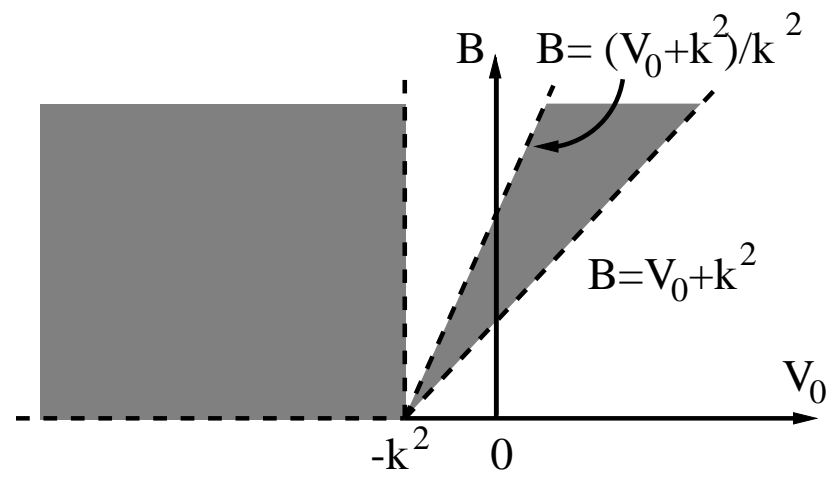

FIG. 2. The region of validity of the solutions of Type A is displayed shaded for a fixed value of $k$. The edges of these regions correspond to various trivial phase solutions. 


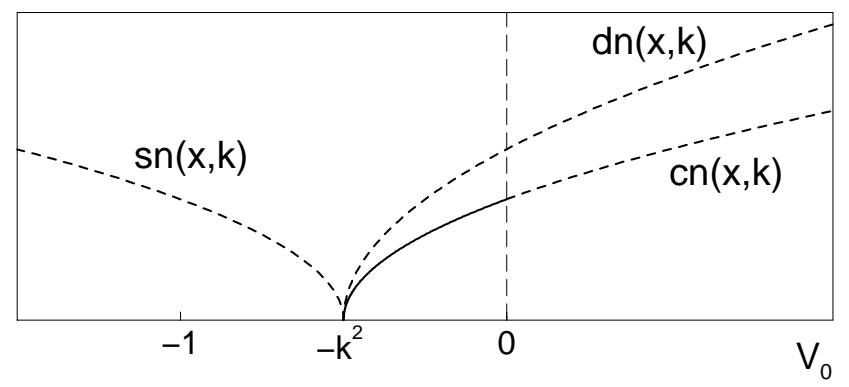

FIG. 3. The amplitude of the trivial phase solutions of Type A versus the potential strength $V_{0}$.

For typical values of $V_{0}, k$ and $B$, the above equations give rise to solutions of Eq. (11) which are not periodic in $x: r(x)$ is periodic with period $2 K(k)$, whereas $\exp (i \theta(x))$ is periodic with period $T=\theta^{-1}(2 \pi)$. In general these two periods $2 K(k)$ and $T$ are not commensurable. Thus, requiring periodic solutions results in another condition, namely $2 K(k) / T=p / q$, for two positive integers $p$ and $q$. The most convenient way to express this phase quantization condition is to assume the potential (i.e., $V_{0}$ and $k$ ) is given, and to consider values of $B$ for which the quantization condition is satisfied. Introducing $\beta=B /\left(V_{0}+k^{2}\right)$, we find

$$
\pm \frac{\sqrt{\beta(\beta-1)\left(1-k^{2} \beta\right)}}{\pi} \int_{0}^{K(k)} \frac{d x}{-\operatorname{sn}(x, k)^{2}+\beta}=\frac{p}{q} .
$$

This equation is solved for $\beta$, after which $B=\beta\left(V_{0}+k^{2}\right)$. For numerical simulations, the number of periods of the potential is set. This determines $q$, limiting the number of solutions of Eq. (9). Solutions with the same periodicity as the potential require $p / q=1$.

Note that solutions of Type A reduce to stationary solutions of Eqs. (11) and (2) with $V_{0}=0$. Furthermore, all stationary solutions of the integrable equation are obtained as limits of solutions of Type A.

\section{Limits and Properties}

The properties of these solutions are best understood by considering their various limit cases.

The trivial phase case: The solutions of Type A have trivial phase when $c=0$. Since $c^{2}$ has three factors which are linear in $B$ (see Eq. (7)), there are three choices of $B$ for which this occurs: $B=0, B=V_{0}+k^{2}$ and $B=\left(V_{0}+k^{2}\right) / k^{2}$. These possibilities are three of the four boundary lines of the region of validity in Fig. 2. Note that the remaining boundary line $\left(V_{0}=-k^{2}\right)$ corresponds to $r^{2}(x)=B$, which gives rise to a plane wave solution. Using Jacobian elliptic function identities [14], one finds that the three other boundary lines give rise to simplified solution forms: $B=0$ gives

$$
\begin{aligned}
& r_{1}(x)=\sqrt{-\left(V_{0}+k^{2}\right)} \operatorname{sn}(x, k), \quad \omega=\frac{1+k^{2}}{2} . \\
B= & V_{0}+k^{2} \text { gives } \\
& r_{2}(x)=\sqrt{V_{0}+k^{2}} \operatorname{cn}(x, k), \quad \omega=\frac{1}{2}-V_{0}-k^{2},
\end{aligned}
$$

where $\operatorname{cn}(x, k)$ denotes the Jacobian elliptic cosine function. Lastly, $B=\left(V_{0}+k^{2}\right) / k^{2}$ gives

$$
r_{3}(x)=\frac{\sqrt{V_{0}+k^{2}}}{k} \operatorname{dn}(x, k), \quad \omega=-1-\frac{V_{0}}{k^{2}}+\frac{k^{2}}{2},
$$

where $\operatorname{dn}(x, k)$ denotes the third Jacobian elliptic function. Solution $(10)$ is valid for $V_{0} \leq-k^{2}$, whereas the other two solutions (11) and (12) are valid for $V_{0} \geq-k^{2}$. The amplitude of these solutions as a function of potential strength $V_{0}$ is shown in Fig. 3 .

Both $\operatorname{cn}(x, k)$ and $\operatorname{sn}(x, k)$ have zero average as functions of $x$ and lie in $[-1,1]$. On the other hand, $\operatorname{dn}(x, k)$ has nonzero average. Its range is $\left[\sqrt{1-k^{2}}, 1\right]$. Furthermore, $\operatorname{cn}(x, k)$ and $\operatorname{sn}(x, k)$ are periodic in $x$ with period $4 K(k)$, whereas $\operatorname{dn}(x, k)$ is periodic with period $2 K(k)$. Some solutions with trivial phase are shown in Fig. 14.

The trigonometric limit: In the limit $k \rightarrow 0$, the elliptic functions reduce to trigonometric functions and $V(x)=-V_{0} \sin ^{2}(x)=\left(V_{0} / 2\right) \cos (2 x)-V_{0} / 2$. Then

$$
r^{2}(x)=-V_{0} \sin ^{2}(x)+B, \quad \omega=\frac{1}{2}-B .
$$

In this case, the phase integral Eq. (1) results in

$$
\tan (\theta(x))= \pm \sqrt{1-V_{0} / B} \tan (x) .
$$

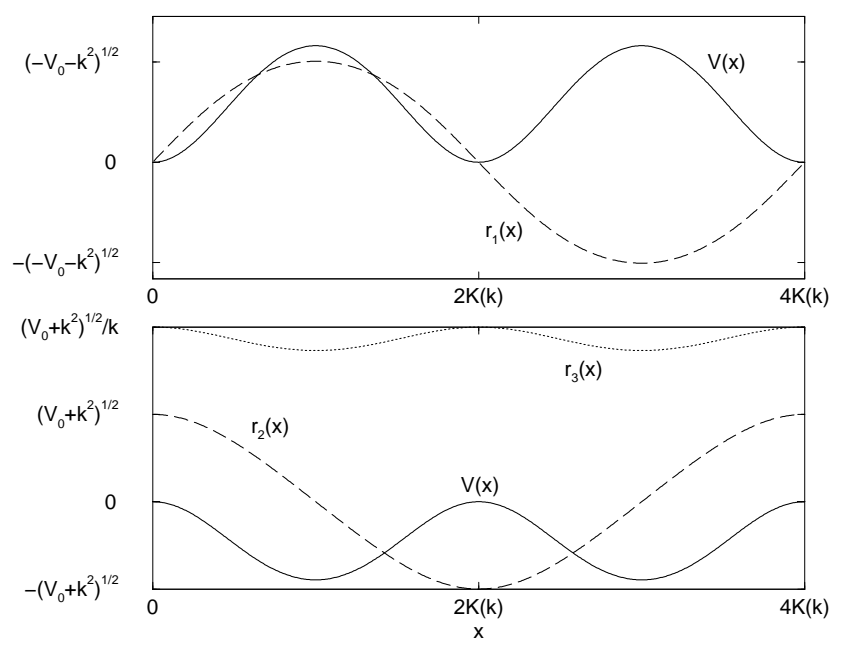

FIG. 4. Trivial phase solutions for $k=0.5 . \quad V(x)$ is indicated with a solid line. For the top figure $V_{0}=-1$. For the bottom figure $V_{0}=1$. 
(a) $\mathrm{V}_{0}=-1, \mathrm{~B}=1$

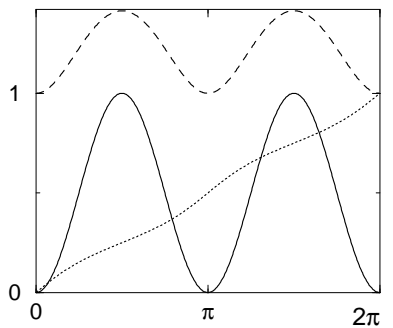

(c) $V_{0}=1, B=3$

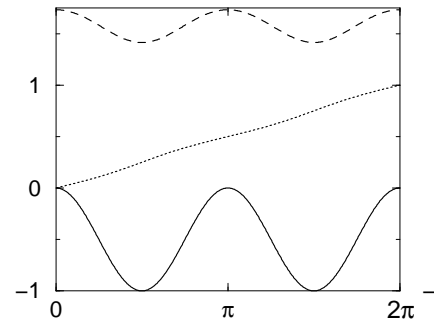

(b) $V_{0}=-1, B=0.1$

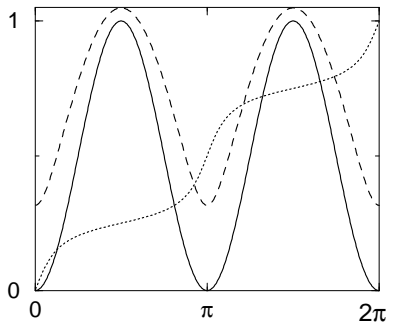

(d) $\mathrm{V}_{0}=1, \mathrm{~B}=1.1$

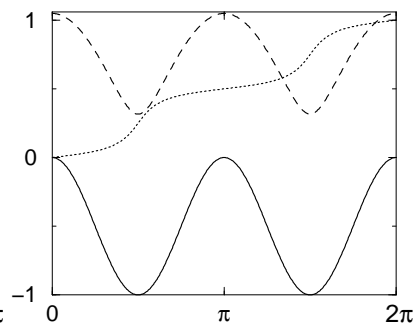

FIG. 5. Phase and amplitude of the trigonometric solutions. For all these figures, the solid line denotes $V(x)$, the dashed line is $r(x)$ and the dotted line is $\theta(x) /(2 \pi)$. Note that $\theta(x)$ becomes piecewise constant, as $B$ approaches the boundary of the region of validity. Far away from this boundary, $\theta(x)$ is essentially linear.

Note that this formula guarantees that the resulting solution is periodic with the same period as the potential, so no phase quantization is required. In the trigonometric limit, the wedge between the two regions of validity in Fig. 2 disappears. This is no surprise, as in this limit, $\operatorname{dn}(x, k) \rightarrow 1$, and the third trivial phase solution reduces to a plane wave solution. The corner point of the region of validity also moves to the origin. Some trigonometric solutions are illustrated in Fig. 5 .

The solitary wave limit: $k=1$. In this limit the elliptic functions reduce to hyperbolic functions. Specifically, $\operatorname{sn}(x, k)=\tanh (x)$. Hence in this limit, the potential has only a single well or a single peak. Then $V_{0}<0$ gives rise to a repulsive potential, whereas $V_{0}>0$ gives rise to an attractive potential: $V(x)=-V_{0} \tanh ^{2}(x)$. In this case the phase $\theta(x)$ of Eq. (任) can be calculated explicitly:

$$
\begin{aligned}
r^{2}(x) & =-\left(V_{0}+1\right) \tanh ^{2}(x)+B \\
\theta(x) & =\sqrt{\frac{-B}{V_{0}+1}} x+\arctan \left(\sqrt{\frac{V_{0}+1}{-B}} \tanh (x)\right),
\end{aligned}
$$

which is valid for $B>0$ and $V_{0}<-1$. This solution is a stationary solitary wave of depression on a positive background. It is reminiscent of the gray soliton solution of the NLS equation with repulsive nonlinearity. Note that this solution exists with an attractive potential. One such solution is illustrated in Fig. 6a. Another solution exists when $B=V_{0}+1>0: r(x)=\sqrt{V_{0}+1} \operatorname{sech}(x)$ and $\theta(x)=0$. This solution represents a stationary elevated solitary wave. It is a deformation of the bright soliton solution of the NLS equation with attractive nonlinearity. Depending on $V_{0}$, it exists in either an attractive $\left(-1<V_{0}<0\right)$ or a repulsive $\left(V_{0}>0\right)$ potential. These solutions are shown in Fig. 6b-c.

Understanding the solitary wave limit facilitates the understanding of what occurs for $k \rightarrow 1$. In this case the solutions of Type A reduce to a periodic train of solitons with exponentially small interactions as illustrated in Fig. 6 d d. The exponentially small nature of the interactions can be exploited analytically as is done in Sec. IV for nonstationary solutions.

\section{Type B}

\section{Derivation}

For these solutions, $r^{2}(x)$ is linear in $\operatorname{sn}(x, k)$ or $\operatorname{dn}(x, k)$. First we discuss the solution with $\operatorname{sn}(x, k)$. The quantities associated with this solution will be denoted with a subindex 1 . The quantities associated with the $\operatorname{dn}(x, k)$ solution receive a subindex 2 .

Substituting

$$
r_{1}^{2}(x)=a_{1} \operatorname{sn}(x, k)+b_{1}
$$

in Eq. (5) and equating different powers of $\operatorname{sn}(x, k)$ gives the relations:

$$
\begin{aligned}
V_{0} & =-\frac{3}{8} k^{2} \\
\omega_{1} & =\frac{1}{8}\left(1+k^{2}\right)-\frac{6 a_{1}^{2}}{k^{2}} \\
c_{1}^{2} & =-\frac{a_{1}^{2}}{4 k^{6}}\left(16 a_{1}^{2}-k^{4}\right)\left(16 a_{1}^{2}-k^{2}\right), \\
b_{1} & =\frac{4 a_{1}^{2}}{k^{2}} .
\end{aligned}
$$

The class of potentials Eq. (22) is restricted by the first of these relations so that $V_{0}$ is in the narrow range $-3 k^{2} / 8 \leq V_{0} \leq 0$. The solution class now depends on one free amplitude parameter $a_{1}$ and the free equation parameter $k$.

The region of validity of this solution is, as before, determined by the requirements $c_{1}^{2} \geq 0$ and $r_{1}^{2}(x) \geq 0$ :

$$
\frac{k}{4} \geq\left|a_{1}\right| \geq \frac{k^{2}}{4}
$$


(a) $\mathrm{V}_{0}=-2, \mathrm{~B}=0.1$

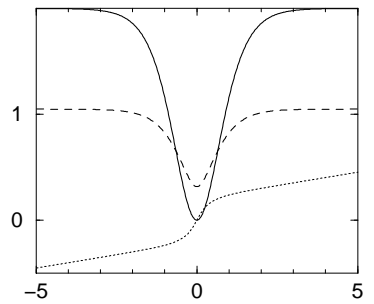

(c) $\mathrm{V}_{0}=1, \mathrm{~B}=1$

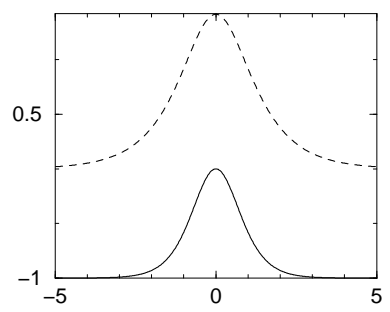

(b) $V_{0}=-0.5, B=1$

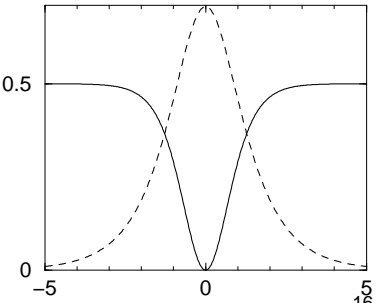

(d) $V_{0}=-2, B=1, k=1-10^{-16}$

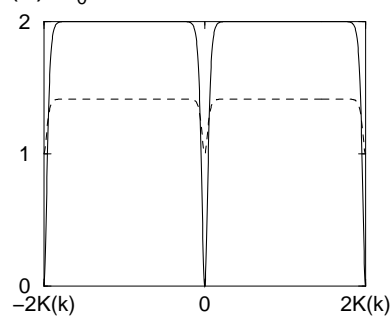

FIG. 6. Solutions with $\mathrm{k}=1$ (a,b,c) or $k \rightarrow 1$ (d). The solid line denotes $V(x)$, the dashed line is $r(x)$ and the dotted line is $\theta(x) / 2 \pi$. In (d), a value of $k=1-10^{-16}$ was used.

The period of $r_{1}(x)$ is twice the period of the potential. Requiring periodicity in $x$ of this first solution of Type B gives

$$
\pm \frac{\sqrt{\left(16 a_{1}^{2}-k^{4}\right)\left(k^{2}-16 a_{1}^{2}\right)}}{4 \pi k^{3}} \int_{0}^{2 K(k)} \frac{d x}{\frac{4 a_{1}}{k^{2}}+\operatorname{sn}(x, k)}=\frac{p}{q} .
$$

For given $k$ and integers $p, q$, this equation is solved for $a_{1}$.

The $\operatorname{dn}(x, k)$ solutions are found by substituting

$$
r_{2}^{2}(x)=a_{2} \operatorname{dn}(x, k)+b_{2},
$$

in Eq. (5). Equating different powers of $\operatorname{dn}(x, k)$ imposes the following constraints on the parameters:

$$
\begin{aligned}
V_{0} & =-\frac{3}{8} k^{2}, \\
\omega_{2} & =\frac{1}{8}\left(1+k^{2}\right)+6 a_{2}^{2}, \\
c_{2}^{2} & =\frac{a_{2}^{2}}{4}\left(16 a_{2}^{2}-1\right)\left(16 a_{2}^{2}+k^{2}-1\right), \\
b_{2} & =-4 a_{2}^{2} .
\end{aligned}
$$

The class of potentials (2) is restricted as for the previous solution by the first of these relations. The solution class again depends on one free amplitude parameter $a_{2}$ and the free equation parameter $k$.

The region of validity of this solution is once more determined by the requirements $c_{2}^{2} \geq 0$ and $r_{2}^{2}(x) \geq 0$ :

$$
0 \leq a_{2} \leq \frac{\sqrt{1-k^{2}}}{4} .
$$

The period of $r_{2}(x)$ is equal to the period of the potential. Requiring periodicity in $x$ of this second solution of Type B gives

$$
\pm \frac{\sqrt{\left(16 a_{2}^{2}-1\right)\left(16 a_{2}^{2}+k^{2}-1\right)}}{\pi} \int_{0}^{K(k)} \frac{d x}{4 a_{2}-\operatorname{dn}(x, k)}=\frac{p}{q} .
$$

For given $k$ and integers $p, q$, this equation is solved to determine $a_{2}$.

In contrast to solutions of Type A, solutions of Type $\mathrm{B}$ do not have a nontrivial trigonometric limit. In fact, for solutions of Type B, this limit is identical to the limit in which the potential strength $V_{0}=-3 k^{2} / 8$ approaches zero. Thus it is clear that the solutions of Type $\mathrm{B}$ have no analogue in the integrable nonlinear Schrödinger equation. However, other interesting limits do exist.

\section{Limits and Properties}

The trivial phase case: Trivial phase corresponds to $c=0$. This occurs precisely at the boundaries of the regions of validity. For the first solution of Type B, there are four possibilities: $a_{1}=k^{2} / 4, a_{1}=k / 4, a_{1}=-k^{2} / 4$ or $a_{1}=-k / 4$. By replacing $x$ by $x+2 K(k)$, one sees that the last two possibilities are completely equivalent to the first two, so only the first two need to be considered. For $a_{1}=k^{2} / 4$,

$$
r_{1}^{2}(x)=\frac{k^{2}}{4}(1+\operatorname{sn}(x, k)), \quad \omega_{1}=\frac{1}{8}-\frac{k^{2}}{4} .
$$

Equating $a_{1}=k / 4$ gives

$$
r_{1}^{2}=\frac{1}{4}(1+k \operatorname{sn}(x, k)), \quad \omega_{1}=-\frac{1}{4}+\frac{1}{8} k^{2} .
$$

For the second solution, there are four possibilities: $a_{2}=$ 0 or $a_{2}=\sqrt{1-k^{2}} / 4$. The first one of these results in a zero solution. The second one gives an interesting trivial phase solutions. Let $k^{\prime}=\sqrt{1-k^{2}}$. Then, for $a_{2}=\sqrt{1-k^{2}} / 4=k^{\prime} / 4$,

$$
r_{2}^{2}(x)=\frac{k^{\prime}}{4}\left(\operatorname{dn}(x, k)-k^{\prime}\right), \quad \omega_{2}=\frac{1}{4}+\frac{k^{\prime 2}}{4} .
$$

These solutions are shown in Fig. 7 . 

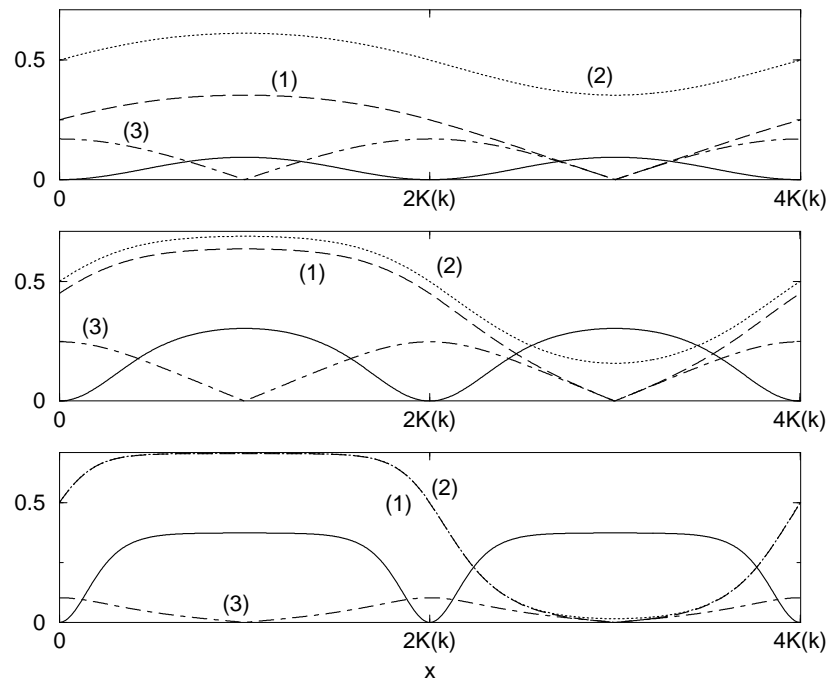

FIG. 7. Solutions of Type B with trivial phase. The figures correspond to, from top to bottom, $k=0.5, k=0.9$ and $k=0.999$. The potential is indicated with a solid line. The other curves are: (1) $\left|r_{1}(x)\right|$ with $a_{2}=k^{2} / 4$, (2) $r_{1}(x)$ with $a_{2}=k / 4$ and (3) $\left|r_{2}(x)\right|$ with $a_{2}=k^{\prime} / 4$.

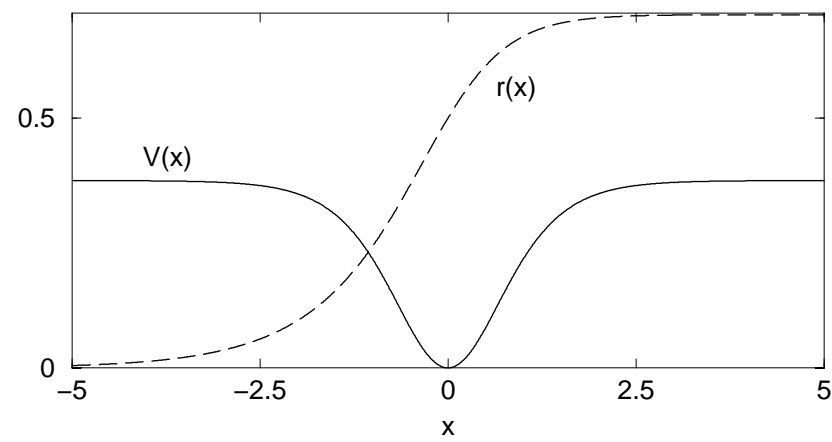

FIG. 8. Solitary wave solution of Type B. The potential is indicated with a solid line. The dashed line solution corresponds to $a=0.3$, the dotted line to $a=-0.3$.

The hyperbolic limit: In this limit $V_{0}=-3 / 8$ and the potential is $V(x)=3 \tanh (x)^{2} / 8$. The region of validity for $r_{2}$ shrinks to $a_{2}=0$, giving only the zero solution. However, the region of validity for $r_{1}$ shrinks to $a_{1}=1 / 4$, giving rise to a non-trivial shock-like solution with trivial phase: $r_{1}^{2}(x)=(1+\tanh (x)) / 4$. This solution is shown in Fig. 8. As for the solutions of type A, the solitary wave limit gives an idea of the behavior of the solution for values of $k \rightarrow 1$.

\section{STABILITY}

We have found a large number of new solutions to the governing Eqs. (11) and (2). However, only solutions that are stable can be observed in experiments. In this section, we consider the stability of the different solutions. Both analytical and numerical results are presented for the solutions with trivial phase. In contrast, only numerical results are discussed for the nontrivial phase cases.

The linear stability of the solution (3) is investigated. To do so, the exact solutions are perturbed by:

$$
\psi(x, t)=(r(x)+\epsilon \phi(x, t)) \exp [i(\theta(x)-\omega t)]
$$

where $\epsilon \ll 1$ is a small parameter. Collecting terms at $O(\epsilon)$ gives the linearized equation. Its real and imaginary parts are $\mathbf{U}=\left(U_{1}, U_{2}\right)^{t}=(\operatorname{Re}[\phi], \operatorname{Im}[\phi])^{t}$ :

$$
\mathbf{U}_{t}=J L \mathbf{U}=J\left(\begin{array}{cc}
L_{+} & S \\
-S & L_{-}
\end{array}\right) \mathbf{U},
$$

where

$$
\begin{aligned}
L_{+} & =-\frac{1}{2}\left(\partial_{x}^{2}-\frac{c^{2}}{r^{4}}\right)-3 r(x)^{2}+V(x)-\omega, \\
L_{-} & =-\frac{1}{2}\left(\partial_{x}^{2}-\frac{c^{2}}{r^{4}}\right)-r(x)^{2}+V(x)-\omega, \\
S & =\frac{c}{r(x)} \partial_{x} \frac{1}{r(x)}
\end{aligned}
$$

and $J=\left(\begin{array}{cc}0 & -1 \\ 1 & 0\end{array}\right)$ is a skew-symmetric matrix. The operator $L$ is Hermitian as are $L_{+}$and $L_{-}$while $S$ is anti-Hermitian. Considering solutions of the form $\mathbf{U}(x, t)=\hat{\mathbf{U}}(x) \exp (\lambda t)$ gives the eigenvalue problem

$$
\mathcal{L} \hat{\mathbf{U}}=\lambda \hat{\mathbf{U}},
$$

where $\mathcal{L}=J L$ and $\lambda$ is complex. If all $\lambda$ are imaginary, then linear stability is established. In contrast, if there is at least one eigenvalue with a positive real part, then instability results. Using the phase invariance $\psi \mapsto e^{i \gamma} \psi$ of Eq. (11), Noether's theorem [16] gives

$$
\mathcal{L}\left(\begin{array}{c}
0 \\
r(x)
\end{array}\right)=0
$$

which implies that $L_{-} r(x)=0$. Thus $\lambda=0$ is in the spectrum of $L_{-}$. For general solutions of the form (3), determining the spectrum of the associated linearized eigenvalue problem (28) is beyond the scope of current methods. However, some cases of trivial-phase solutions $(c=0)$ are amenable to analysis.

The Hermitian operators $L_{ \pm}$are periodic Schrödinger operators and thus the spectra of these operators is real and consists of bands of continuous spectrum contained in $\left[\lambda_{ \pm}, \infty\right)$ [16]. Here $\lambda_{ \pm}$denote the ground state eigenvalues of $L_{ \pm}$respectively. They are given by

$$
\lambda_{ \pm}=\inf _{\|\phi\|=1}\left\langle\phi\left|L_{ \pm}\right| \phi\right\rangle
$$


where $\|\phi\|^{2}=\langle\phi \mid \phi\rangle$. From the relation $L_{+}=L_{-}-2 r(x)^{2}$ it follows that $\lambda_{+}<\lambda_{-}$. Also $\lambda_{-} \leq 0$ since $\lambda=0$ is an eigenvalue of $L_{-}$.

If $\lambda_{-}=0$, then $L_{-}$is non-negative and self-adjoint, so we can define the non-negative square root, $L_{-}^{\frac{1}{2}}$, via the spectral theorem [16], and hence the Hermitian operator $H=L_{-}^{\frac{1}{2}} L_{+} L_{-}^{\frac{1}{2}}$ can be constructed. The eigenvalue problem for $\mathcal{L}$ in Eq. (30) is then equivalent to

$$
\left(H+\lambda^{2}\right) \varphi=0,
$$

with $\hat{U}_{1}=L_{-}^{\frac{1}{2}} \varphi$. Denote the left-most point of the spectrum of $H$ by $\mu_{0}$. If $\mu_{0} \geq 0$ then $\lambda^{2}<0$ and the eigenvalues of $\mathcal{L}$ are imaginary and linear stability results. Since $H=L_{-}^{\frac{1}{2}} L_{+} L_{-}^{\frac{1}{2}}$ and $L_{-}^{\frac{1}{2}}$ is positive, $\mu_{0} \geq 0$ if and only if $L_{+}$is non-negative. In contrast, if $\mu_{0}<0$ then $\lambda^{2}>0$ and $\mathcal{L}$ has at least one pair of real eigenvalues with opposite sign. This shows the existence of a growing mode leading to instability of the solution.

This non-perturbative method distinguishes between two cases

A. If $r(x)>0$ then Eq. (31) implies $r(x)$ is the ground state of $L_{-}$so that $\lambda_{-}=0$ [16] while $\lambda_{+}<0$. Thus the solution (3) is unstable.

B. If $r(x)$ has a zero, it is no longer the ground state [16] and $\lambda_{-}<0$. Thus $\lambda_{-}$and $\lambda_{+}$are both negative and the situation is indefinite. The nonperturbative methods are insufficient to determine linear stability or instability.

While the solutions in Eqs. (12) and (25) satisfy the instability criterion, the stability of the remaining solutions is undetermined by this method. We therefore rely on direct computations of the nonlinear governing Eqs. (1) and (2) to determine stability. Where the numerics indicate stability, perturbative methods are used to confirm this observation. For all computational simulations, twelve spatial periods are used. However, to better illustrate the dynamics, typically four spatial periods are plotted. Moreover, all computations are performed with white noise included in the initial data.

\section{A. trivial phase: Type A}

$$
\text { 1. } d n(x, k)
$$

In the case of the $\operatorname{dn}(x, k)$ solution (12), $r(x)>0$ and the instability criterion A applies. The unstable evolution is depicted in Figs. 9 and 10. With $V_{0}=-0.3$ and $k=0.7$ the onset of instability occurs at $t \approx 20$. Figure 10 depicts an overhead view of the dynamics with the onset of instability for $k=0.7$ and for values of the potential strength $V_{0}=-0.3,0,0.3$. For increasing values of the amplitude, $A=\sqrt{V_{0}+k^{2}} / k$, the onset of

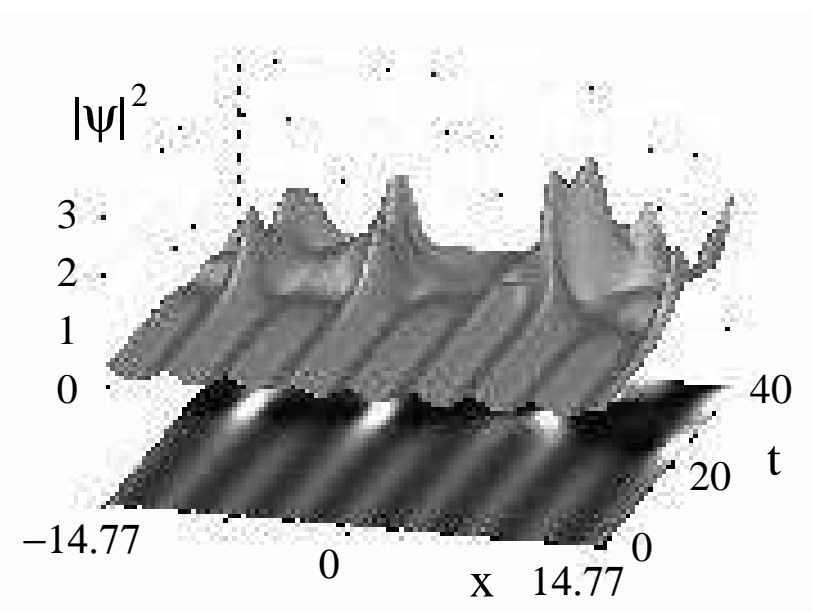

FIG. 9. Unstable evolution of a Type $\mathrm{A} \operatorname{dn}(x, k)$ solution given by Eq. (12) over 40 time units with $k=0.7$ and $V_{0}=-0.3$.

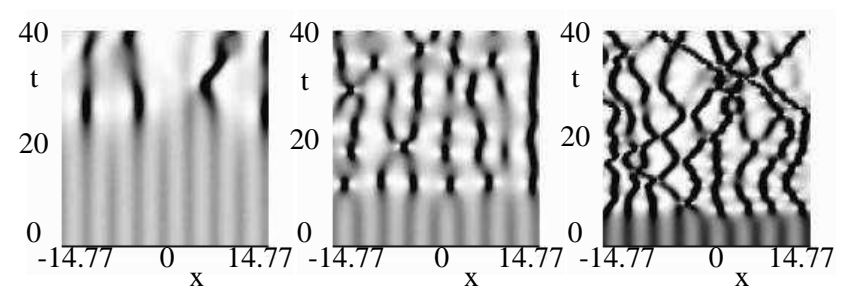

FIG. 10. Top view of the unstable evolution of Type A $\operatorname{dn}(x, k)$ solutions given by Eq. (12) over 40 time units with $k=0.7$ and with $V_{0}=-0.3$ (left), $V_{0}=0.0$ (center), and $V_{0}=0.3$ (right).

instability occurs earlier. For the middle figure of Fig. 10, $V_{0}=0$ and we are solving the integrable nonlinear Schrödinger equation. The instability of this solution is not surprising since $\operatorname{dn}(x, k)$ resembles a plane wave which is known to be modulationally unstable [15]. For increasing values of the amplitude $A$, the nonlinearity in (11) becomes more dominant, resulting in an earlier onset of the modulational instability.

$$
\text { 2. } \operatorname{sn}(x, k)
$$

For the $\operatorname{sn}(x, k)$ solution (10), the indeterminate stability criterion B results. Stability analysis for the linear Schrödinger equation suggests that this case is unstable since the density is localized on the peaks of the potential (see Fig. 1). Figure 11 illustrates this behavior and shows the onset of instability to occur for $t \approx 20$. 


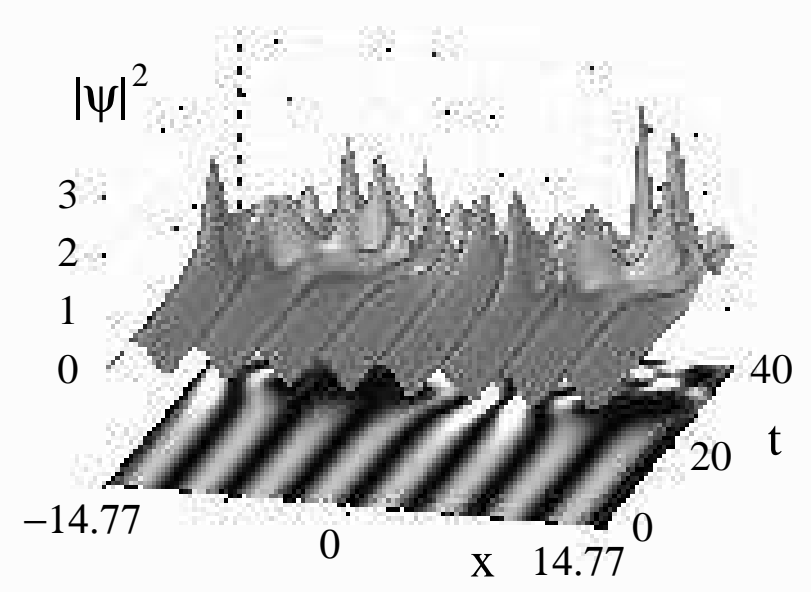

FIG. 11. Unstable evolution of a Type A $\operatorname{sn}(x, k)$ solution given by Eq. (10) over 40 time units with $k=0.7$ and $V_{0}=-1.0$.

\section{3. $c n(x, k)$}

For the $\operatorname{cn}(x, k)$ solution (11), the indeterminate stability criterion B results once again. However, now the stability analysis for the linear Schrödinger equation suggests that two distinct cases must be considered. For $V_{0}>0$, the density is localized on the peaks of the potential, and the solution is unstable as illustrated in the bottom of Fig. 12. The onset of instability occurs near $t \approx 30$. In contrast, for $-k^{2}<V_{0}<0$, the density is localized in the troughs of the potential which suggests that the solution might be stable. Indeed, as the top of Fig. 12 illustrates, the $\operatorname{cn}(x, k)$ solution is stable in this regime.

\section{B. Trivial Phase: Type B}

$$
\text { 1. } \operatorname{sn}(x, k)
$$

For the Type B $\operatorname{sn}(x, k)$ solution (24) with $a_{1}=k^{2} / 4$, the indeterminate stability criterion $\mathrm{B}$ applies. For this solution, the period of the density is twice the period of the potential so that the density is not localized in the troughs of the potential. Stability analysis for the linear Schrödinger equation suggests that this case is unstable as with the Type A $\operatorname{sn}(x, k)$ solution. Figure 13 illustrates this behavior and shows the onset of instability to occur for $t \approx 200$ for $k=0.5$ and $t \approx 15$ for $k=0.999$.

The Type B $\operatorname{sn}(x, k)$ solution (24) with $a_{1}=k / 4$ is nodeless. Thus $r(x)>0$ and the instability criterion $\mathrm{A}$ results. Figure 14 illustrates this behavior and shows the onset of instability to occur at $t \approx 20$ for $k=0.5$.
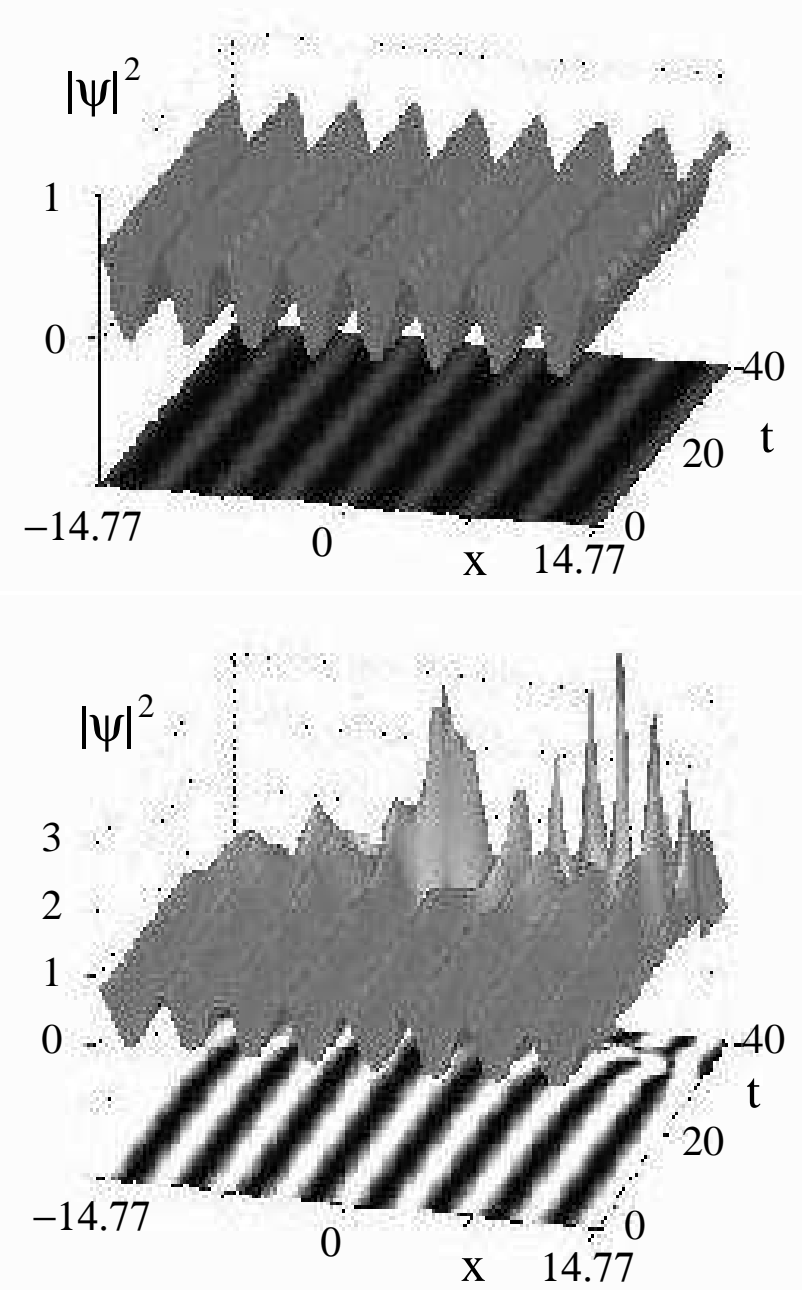

FIG. 12. Dynamics of Type A cn $(x, k)$ solutions given by Eq. (11) over 40 time units with $k=0.7$ and with $V_{0}=-0.3$ (top, stable) and $V_{0}=0.3$ (bottom, unstable).

$$
\text { 2. } \operatorname{dn}(x, k)
$$

For the Type $\mathrm{B} \operatorname{dn}(x, k)$ solution (26), the indeterminate stability criterion B results again. However, the stability analysis for the linear Schrödinger equation again suggests that the solution might be stable since the density is localized in the troughs of the potential. The dynamics of this solution for $k=0.5$ is illustrated in Fig. 15 .

\section{NONSTATIONARY SOLUTIONS: SPATIALLY EXTENDED BREATHERS}

Equation (1) has many solutions describing condensates that oscillate in time. In this section, we construct such space and time periodic solutions in the large well separation limit $(k \rightarrow 1)$. We consider only time-periodic solutions for which the condensate in each potential well 

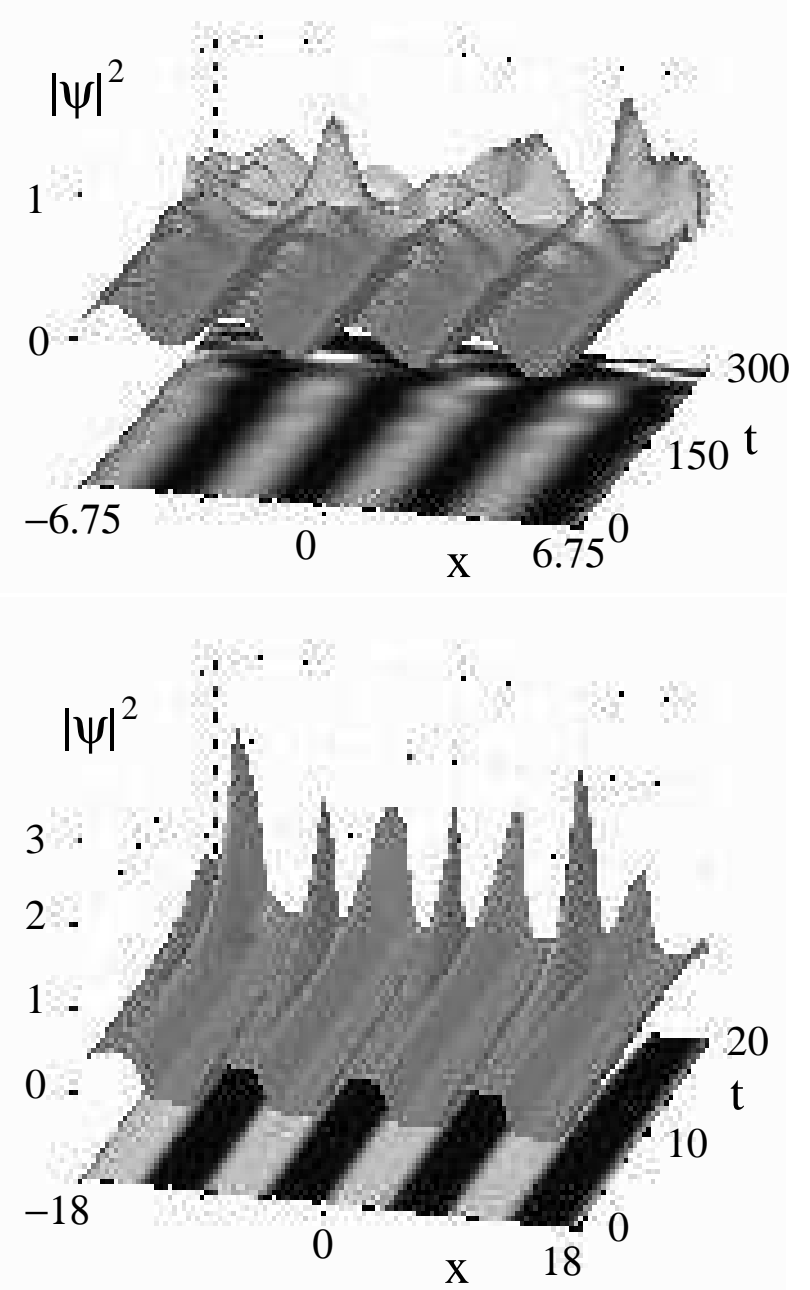

FIG. 13. Unstable evolution of Type B $\operatorname{sn}(x, k)$ solutions with $a_{1}=k^{2} / 4$ given by Eq. (24) over 300 time units for $k=0.5$ (top) and over 20 time units for $k=0.999$.

oscillates with the same frequency. More time-periodic solutions will be considered elsewhere [17].

Our solutions are obtained through a series of approximations. First, we assume the ansatz

$$
\psi(x, t)=A \exp (-i \omega t) \sum_{k=-\infty}^{\infty} \operatorname{sech}\left(x-\xi_{k}(t)\right) .
$$

This equation is motivated by the fact that a trivial-phase $\operatorname{cn}(x, k \rightarrow 1)$-solution can be written as $\sum_{j=-\infty}^{\infty} \operatorname{sech}(x-$ $4 j K(k))$ up to exponentially small terms describing the interaction between neighboring peaks. Note that the amplitude $A$ and frequency $\omega$ in Eq. (34) are fixed across the condensate.

Using the variational Lagrangian reduction approach 18], effective equations for the motion of a single lump of condensate are derived. The dynamics of the center of a lump of condensate $\xi_{k}(t)$ are then given by

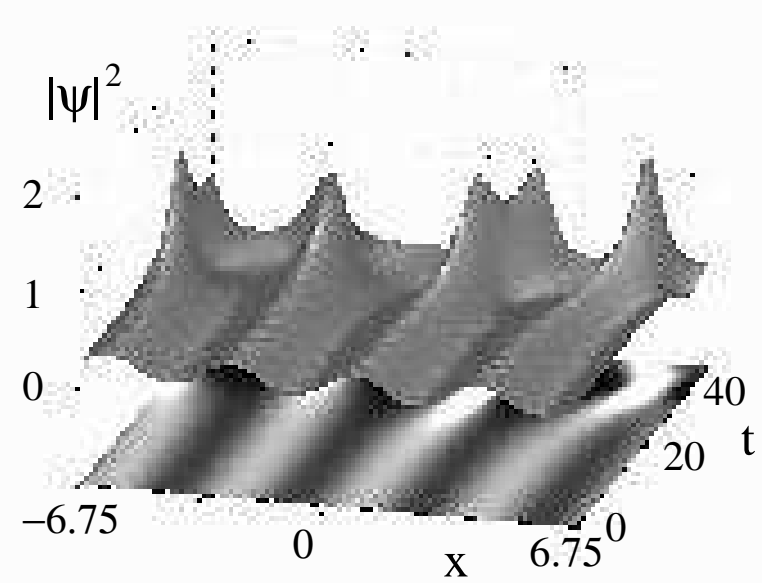

FIG. 14. Unstable evolution of a Type B $\operatorname{sn}(x, k)$ solution with $a_{1}=k / 4$ given by Eq. (25) over 40 time units with $k=0.5$.

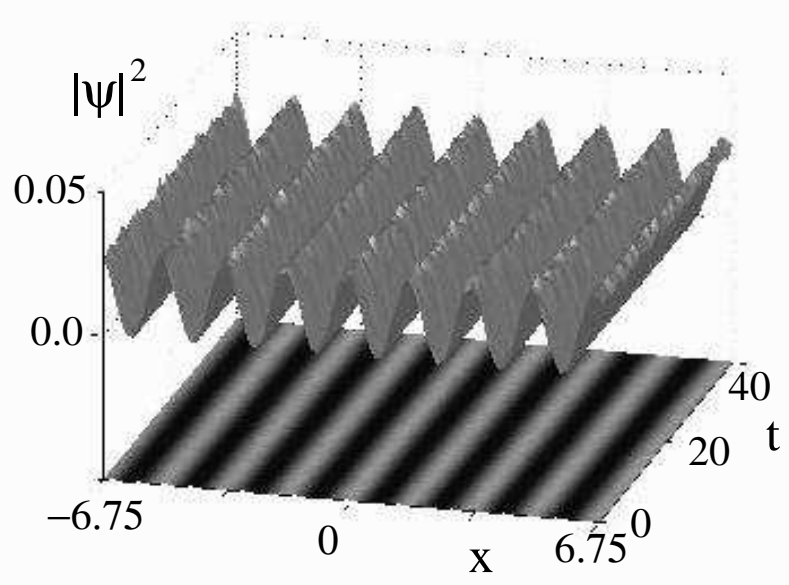

FIG. 15. Stable evolution of the Type B $\operatorname{dn}(x, k)$ solution given by Eq. 26) over 40 time units with $k=0.5$.

the Newtonian equation of motion, $\ddot{\xi}_{k}=-\partial W\left(\xi_{k}-\right.$ $\left.\xi_{k}^{0}\right) / \partial \xi_{k}$ where $\xi_{k}^{0}$ is the center position of the $k$ th potential well, with potential

$$
W(\zeta)=-V_{0} \bar{\nu} \zeta^{2}\left(\alpha-\beta \zeta^{2}+O\left(\zeta^{3}\right)\right)
$$

where $\bar{\nu}$ is the average width of the condensate [17], $\alpha=$ $4 / 15$ and $\beta=2 / 63$. For a small displacement the condensate undergoes near-harmonic oscillations with frequency $\sqrt{-2 \alpha V_{0} \bar{\nu}}$

After the self-interaction, the next largest contributions are the nearest-neighbor interactions which arise from exponentially small tail overlaps. These interactions result in the following lattice differential equation (LDE) for the $k$ th position $\xi_{k}(t)$, 


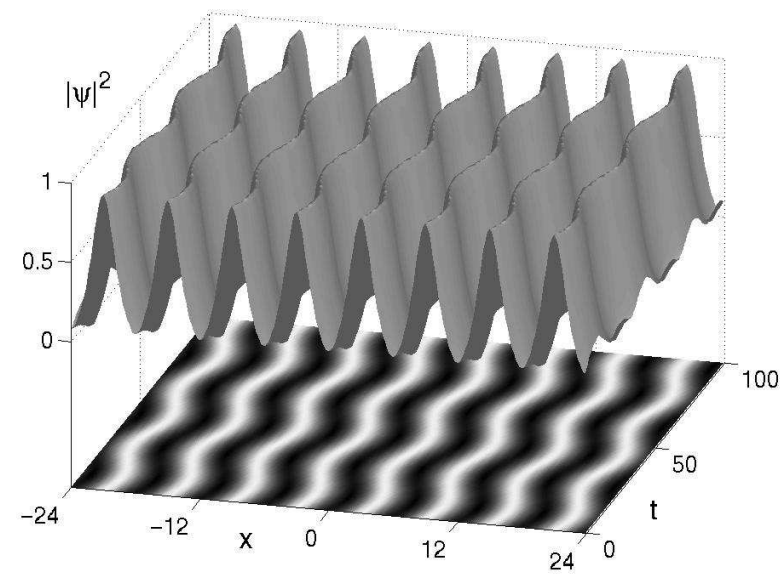

FIG. 16. Stable vibrational mode of the condensate corresponding to the fixed point $P_{2}$ over 100 time units with $V_{0}=-0.1$. The initial conditions are a perturbation of the cnoidal wave solution (11) with $k=0.9791048444$ giving an initial lump-to-lump separation of $\Delta \xi_{k}=6$.

$$
\ddot{\xi}_{k}=-4 A^{3}\left(e^{-A \Delta \xi_{k}}-e^{-A \Delta \xi_{k-1}}\right)+W^{\prime}\left(\xi_{k}-\xi_{k}^{0}\right),
$$

Here $\Delta \xi_{k}=\xi_{k+1}-\xi_{k}$ is the separation between centers of consecutive condensates. The same LDE can be derived from soliton perturbation theory [19,20] or a variational approach [21] and corresponds to a Toda lattice 22] with additional on-site potentials due to $W$.

We look for oscillatory solutions of Eq. (36) by considering a Fourier expansion for $\xi_{k}$ [23]:

$$
\xi_{k}(t)=\sum_{j=0}^{\infty} a_{k}(j) \cos (j \Omega t) .
$$

We insert this ansatz into Eq. (36) and Taylor expand the exponentials, keeping terms to second order. Equating coefficients of $\cos (j \Omega t)$, we obtain a recurrence relation between the amplitudes $a_{k}=a_{k}(j=1)$ :

$$
a_{k+1}=3 \beta W_{0} a_{k}^{3}+\left(2+4 \alpha W_{0}-\bar{\Omega}^{2}\right) a_{k}-a_{k-1},
$$

where by using $\left|\Delta \xi_{k}^{0}\right|=2 K(k)$ we find $\bar{\Omega}=$ $\Omega \exp (A K(k)) / 2 A^{2}$ and $W_{0}=\left(V_{0} / 32 A^{3}\right) \exp (2 A K(k))$.

The recurrence relation Eq. (38) may be written as a two dimensional map 24] $\left(x_{k+1}, y_{k+1}\right)=F\left(x_{k}, y_{k}\right)$ by defining $x_{k}=a_{k}$ and $y_{k}=a_{k-1}$ :

$$
\begin{aligned}
& x_{k+1}=3 \beta W_{0} x_{k}^{3}+\left(2+4 \alpha W_{0}-\bar{\Omega}^{2}\right) x_{k}-y_{k} \\
& y_{k+1}=x_{k} .
\end{aligned}
$$

Fixed points of this map are calculated by solving $\left(x_{0}, y_{0}\right)=F\left(x_{0}, y_{0}\right)$. This results in three fixed points $P_{i}=\left(x_{0}, y_{0}\right)(i=1,2,3): P_{1}=(0,0), P_{2}=\left(x^{*}, y^{*}\right)$, and $P_{3}=\left(-x^{*},-y^{*}\right)$ where

$$
x^{*}=y^{*}= \pm \sqrt{\frac{\bar{\Omega}^{2}-4 \alpha W_{0}}{3 \beta W_{0}}},
$$

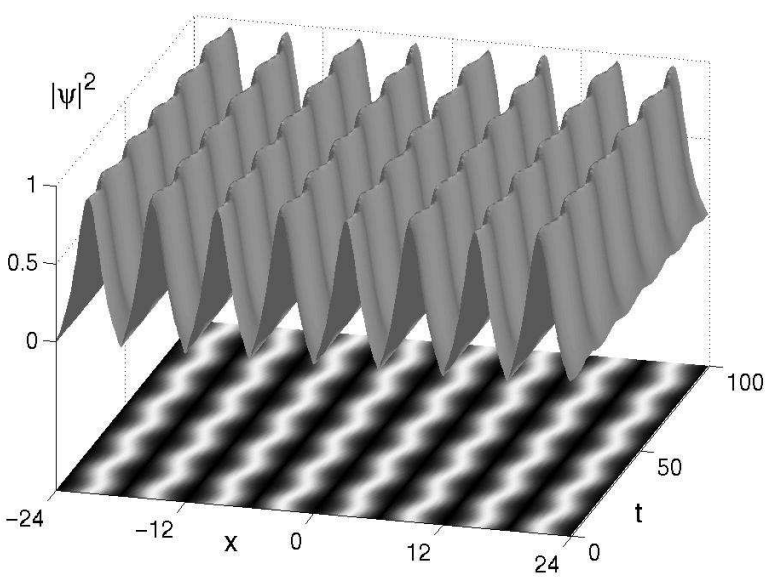

FIG. 17. Stable two-period vibrational mode of the condensate corresponding to a period-two orbit over 100 time units with $V_{0}=-0.1$. The initial conditions are a perturbation of the cnoidal wave solution (11) with $k=0.9791048444$ giving an initial lump-to-lump separation of $\Delta \xi_{k}=6$.

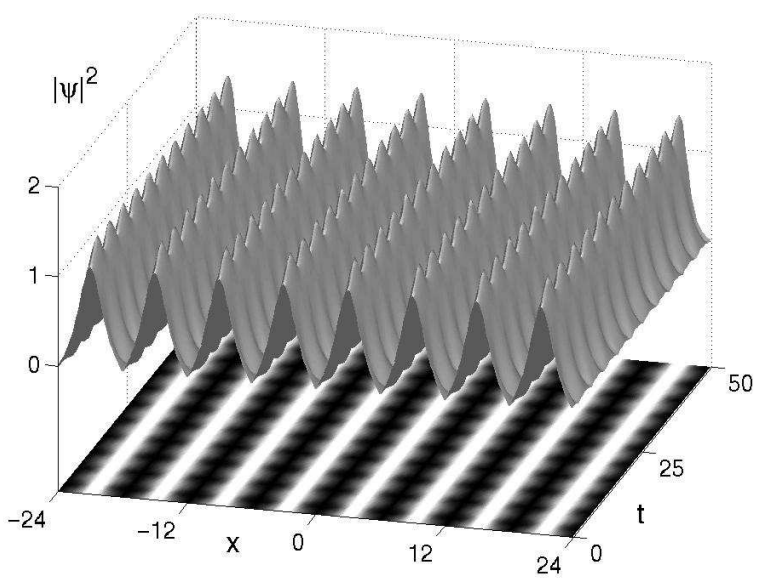

FIG. 18. Stable evolution of a breathing mode of the condensate over 50 time units with $V_{0}=-0.1$. The initial conditions are a perturbation of the cnoidal wave solution (11) with $k=0.9791048444$ giving an initial lump-to-lump separation of $\Delta \xi_{k}=6$.

provided the root is real. From Eq. (37), fixed points $P_{2}$ and $P_{3}$ correspond to vibrational modes in which each lump of condensate oscillates in time with the same amplitude, frequency $\Omega$, and phase. This dynamic is shown in Fig. 16 which is obtained from numerical simulation of Eq. (11). 
Period-two orbits of this map are calculated by solving $\left(x_{0}, y_{0}\right)=F\left(F\left(x_{0}, y_{0}\right)\right)$ and are of the form $\{(\hat{x}, \hat{y}),(-\hat{x},-\hat{y})\}$ 24]. This period two orbit corresponds to two alternating amplitudes $(\hat{x}$ and $-\hat{x})$ of oscillation for consecutive condensates. Since the period two orbit is symmetric with respect to the origin, consecutive condensates oscillate with the same magnitude but opposite phase. The dynamics for this case are show in Fig. 17 .

In a similar fashion, period-three and higher behavior can also be analyzed with the given ansatz Eq. (34). In addition to allowing the lump positions to vary in time, we can also capture amplitude time variations. For simplicity, we illustrate the case where only the amplitudes vary in time. The construction of the appropriate LDE follows from previous methods. The fixed-point solution is illustrated in Fig. 18 where lumps of condensate oscillate in time with the same time-periodic amplitude, frequency, and phase. This solution type is referred to as an extended breather. Spatially localized breathers also exist and will be considered elsewhere 17

\section{SUMMARY AND CONCLUSIONS}

We considered the attractive nonlinear Schrödinger equation with an elliptic function potential as a model for a trapped, quasi-one-dimensional Bose-Einstein condensate. Two new families of periodic solutions of this equation were found and their stability was investigated both analytically and numerically. Additionally, stable time-periodic solutions have been analyzed.

Using perturbations with trivial phase (analysis) or perturbations with random phase (numerics), we find that stationary trivial-phase solutions are stable provided they have nodes and their density is localized in the troughs of the potential. Nodeless solutions are unstable with respect to this same class of perturbations. This is reminiscent of the modulational instability of the plane wave solution of the attractive integrable nonlinear Schrödinger equation.

Using random-phase perturbations, we find all nontrivial-phase solutions to be unstable. However, the time scale for the onset of instability for nontrivial-phase solutions varies significantly: nontrivial-phase solutions with parameter values close to values for trivial-phase solutions appear unaffected by the perturbation for long times. Other nontrivial-phase solutions go unstable more quickly. This implies that even stable trivial-phase solutions are structurally unstable: the smallest amount of phase ramping causes such solutions to lose their stability, albeit on time scale which may be longer than the lifetime of the BEC.

This result implies self-focusing of any attractive stationary condensate. However, the effects of self-focusing can be negligible on the lifetime of the BEC if there is no phase ramping, the density is localized in the wells of the potential, and adjacent density peaks are separated by nodes. Therefore, we have demonstrated within the mean field model the existence of at least one experimentally stable stationary state of an attractive BEC trapped in a standing light wave. In addition, some timeperiodic condensates offer an alternative to experimental stabilization in a standing light wave potential.

Acknowledgments: We benefited greatly from discussions with William Reinhardt. The work of J. Bronski, L. D. Carr, B. Deconinck, and J. N. Kutz was supported by National Science Foundation Grants DMS9972869, CHE97-32919, DMS-0071568, and DMS9802920 respectively. K. Promislow acknowledges support from NSERC- 611255

* to whom correspondence should be addressed

[1] S. L. Cornish et al., e-print cond-mat/0004290 (2000) and references therein.

[2] W. Ketterle, D. S. Sturfee, and D. M. Stamper-Kurn, in Proceedings of the International School of Physics "Enrico Fermi" (IOS Press, Amsterdam; Washington, D.C. 1999), pp. 67-176.

[3] C. C. Bradley, C. A. Sacket, J. J. Tollett, and R. G. Hulet, Phys. Rev. Lett. 75, 1687 (1995).

[4] C. C. Bradley, C. A. Sacket, and R. G. Hulet, Phys. Rev. A 55, 3951 (1997).

[5] C. A. Sackett, J. M. Gerton, M. Welling, and R. G. Hulet, Phys. Rev. Lett. 82, 876 (1999).

[6] L. D. Carr, M. A. Leung, and W. P. Reinhardt, J. Phys. B 33 (2000).

[7] G. Baym, Lectures in Quantum Mechanics, (AddisonWesley, Redwood City, CA), Ch. 20.

[8] L. P. Pitaevskii, Sov. Phys. JETP 13, 451 (1961).

[9] E. P. Gross, Nuovo Cimento 20, 454 (1961).

[10] L. D. Carr, C. W. Clark, and W. P. Reinhardt, Phys. Rev. A 62, 0436XX-1 (2000).

[11] D. S. Petrov, M. Holzmann, and G. V. Shylapnikov, Phys. Rev. Letts. 84, 2551 (2000).

[12] F. Dalfovo, S. Giorgini, L. P. Pitaevskii, and S. Stringari, Rev. Mod. Phys. 71, 463 (1999).

[13] M. Key et al., Phys. Rev. Letts. 84, 1371 (2000); N. H. Dekker et al., Phys. Rev. Letts. 84, 1124 (2000).

[14] Handbook of Mathematical Functions, edited by M. Abramowitz and I. A. Stegun (National Bureau of Standards, Washington, D. C. 1964).

[15] Algebro-geometric approach to nonlinear integrable equations, E. D. Belokolos, A. I. Bobenko, V. Z. Enol'skii, A. R. Its and V. B. Matveev (Springer-Verlag, Berlin, 1994).

[16] R. Courant and D. Hilbert, Methods of Mathematical Physics, (Wiley, New York, 1989).

[17] R. Carretero-González and K. Promislow., Discrete and 
Continuous Dynamical Systems (Series B), to be submitted 2000.

[18] V.M. Pérez-Garcia, H. Michinel, J.I. Cirac, M. Lewenstein and P. Zoller., Phys. Rev. Lett. 77, 5320 (1996).

[19] V.I. Karpman and V.V. Solov'ev, A perturbation theory for soliton systems, Physica D 3, 142 (1981).

[20] V.S. Gerdjikov, I.M. Uzunov, E.G. Estaviev and G.L. Diankov, Phys. Rev. E 55, 6039 (1997).
[21] J. M. Arnold, Phys. Rev. E, 60, 979 (1999).

[22] M. Toda, Theory of Nonlinear Lattices., (SpringerVerlag, New York, 1989).

[23] T. Bountis, H.W. Capel, M. Kollmann, J.C. Ross, J.M. Bergamin, J.P. van der Weele, Phys. Lett. A 268, 50 (2000).

[24] A. J. Lichtenberg and M. A. Lieberman, Regular and Chaotic Dynamics, (Springer-Verlag, New York, 1992). 